PROCEEDINGS OF THE

AMERICAN MATHEMATICAL SOCIETY

Volume 139, Number 12, December 2011, Pages 4467-4473

S 0002-9939(2011)10872-4

Article electronically published on April 26, 2011

\title{
ISOPERIMETRIC SURFACES WITH BOUNDARY
}

\author{
REBECCA DORFF, DREW JOHNSON, GARY R. LAWLOR, AND DONALD SAMPSON
}

(Communicated by Jianguo Cao)

\begin{abstract}
We prove that many common combinations of soap films and soap bubbles that result from dipping polyhedral wire frames in a soap solution are minimizing with respect to their boundary and bubble volume. This can be thought of as a combination of the Plateau problem of least area for surfaces spanning a given boundary and the isoperimetric problem of least area for surfaces enclosing a given volume. Proof is given in arbitrary dimension using a combination of the mapping of Gromov, after Knothe, and the paired calibrations of Lawlor and Morgan.
\end{abstract}

In this paper, we prove that many common soap-film-like surfaces that enclose a volume and have a regular polytope "wireframe" as boundary are mass minimizing with respect to their boundary and enclosed volume. Examples of such surfaces are shown in Figure 1. We give a direct proof using the flux of specialized vector fields to model the mass of each competitor a la Gromov [5] and Lawlor and Morgan 8 .

We propose the name of "equitent problem" for an area minimization question in which competitors must enclose a given volume ("equal content") as well as span a given boundary ("equal extent"). Equitent problems are thus a generalization of Plateau problems (fixed boundary) and isoperimetric problems (fixed enclosed volume). The equitent problem appears largely unexplored to date; we give what we believe to be the first major results.

\section{Knothe-Gromov}

In 1989 Mikhail Gromov 5, following work of Herbert Knothe 7, described a beautiful method for proving isoperimetric theorems. He used what is now called the Knothe-Rosenblatt rearrangement, developed independently by Knothe [7] and Rosenblatt [10], which we now describe. Construct an area-preserving map $F$ from a competitor $U$ to the round ball $B$ of radius $r$ (centered at the origin) of the same volume by sending 'slices' of $U$ to 'slices' of $B$, and repeating the same process in each slice. Specifically, let this map be given by

$$
\begin{gathered}
F: U \rightarrow B \\
\left(y_{1}, y_{2}, \ldots, y_{n}\right) \mapsto\left(z_{1}, z_{2}, \ldots, z_{n}\right),
\end{gathered}
$$

Received by the editors October 8, 2010 and, in revised form, October 20, 2010.

2010 Mathematics Subject Classification. Primary 53C38; Secondary 49Q10.

Key words and phrases. Metacalibration, calibration, isoperimetric, equitent. 
such that

$$
\begin{aligned}
& \frac{\mathcal{H}^{n-j+1} U \cap\left\{x_{1}=y_{1}, \ldots, x_{j-1}=y_{j-1}, x_{j} \leq y_{j}\right\}}{\mathcal{H}^{n-j+1} U \cap\left\{x_{1}=y_{1}, \ldots, x_{j-1}=y_{j-1}\right\}} \\
= & \frac{\mathcal{H}^{n-j+1} B \cap\left\{x_{1}=z_{1}, \ldots, x_{j-1}=z_{j-1}, x_{j} \leq z_{j}\right\}}{\mathcal{H}^{n-j+1} B \cap\left\{x_{1}=z_{1}, \ldots, x_{j-1}=z_{j-1}\right\}}
\end{aligned}
$$

for all $1 \leq j \leq n$. Thus $F_{j}$ is seen to depend only on the variables $\left\{x_{1}, x_{2}, \ldots, x_{j}\right\}$. Scaled volume is preserved set-wise under each 'slice,' with the last coordinate mapped (locally) linearly on the final 'slice' of a portion of a line in $U$ mapped to a line segment in $B$. Volume is then preserved pointwise. In addition, if $U=B$, then $F$ is simply the identity on $B$.

This scheme makes $D F$ lower triangular with positive diagonal entries. Since $\prod_{i=1}^{n} \frac{\partial F_{i}}{\partial x_{i}}=\operatorname{det} D F=1$, the arithmetic-geometric mean inequality implies that $\operatorname{tr} D F \geq n$. Thinking of $F$ as a vector field on $U, \operatorname{tr} D F=\operatorname{div} F \geq n$. Now since the image of $F$ is a ball of radius $r$, the length of the vector $\frac{1}{r} F(\mathbf{x})$ is always at most 1 . The divergence theorem then gives us that

$$
\mathcal{H}^{n-1} \partial U=\int_{\partial U} 1 \geq \int_{\partial U} \frac{1}{r} F \cdot \mathbf{n}=\int_{U} \frac{1}{r} \operatorname{div} F \geq \frac{n}{r} \cdot \mathcal{H}^{n} U,
$$

with equality for the ball. The standard isoperimetric inequality in $\mathbb{R}^{n}$ results.

If we replace $B$ with any convex set, this scheme still works to create a volume preserving map $F$ with divergence at least $n$. The details of this construction were worked out by Brothers and Morgan in [2]. They also provide a version of the divergence theorem with weakened hypotheses and show that $F$ satisfies these.

\section{PAIRED CALIBRATION}

In 1993, Lawlor and Morgan [8] gave a simple proof that in every dimension, the cone over the $(n-2)$-skeleton of the regular simplex is area-minimizing. In $\mathbb{R}^{2}$ this is the length-minimizing $Y$-shaped figure of three edges meeting at 120-degree angles, and in $\mathbb{R}^{3}$ it is the union of isosceles triangles from each edge of a regular tetrahedron into its center of mass. This result had already been proved by Jean Taylor in 1975 [1] for $\mathbb{R}^{3}$. However, the result by Lawlor and Morgan was new in higher dimensions.

A simple description of their "paired calibration" technique is to line up (in $\mathbb{R}^{3}$, say) four heat lamps shining directly toward the faces of a regular tetrahedron. Consider that the heat rays from a lamp are parallel with constant intensity, and are completely absorbed when they hit a surface. Now place the proposed minimizing cone in this configuration for a set time, and measure the temperature at each point of the cone. Replace the cone with any competing surface for the same amount of time, and measure its temperature. Because each piece of the cone has the ideal surface angle at which to absorb heat from the two lamps that shine on that piece (analogous to facing a single lamp at 90 degrees), we know that the cone is at least as hot, pointwise, as the competing surface. On the other hand, because both competitors span the same boundary, the total heat absorbed is the same for both. Equal heat absorbed and hotter temperature pointwise means the cone must have less surface area over which to distribute the heat. This approach is made rigorous by representing heat absorption by the flux integrals of a set of constant vector fields. 
We will combine the Knothe-Gromov vector field approach with paired calibration.

\section{THE SURfACES}

A uniform polytope in $\mathbb{R}^{n}$ (or uniform polyhedron in $\mathbb{R}^{3}$ ) is a polytope with symmetric vertices made of uniform polytope facets of one dimension down. The uniform polytopes in two dimensions are the regular polygons. In particular the edges of a uniform polytope are all of equal length, and the vertices of a uniform polytope all have equal distance from its center of mass. Examples of uniform polyhedra in $\mathbb{R}^{3}$ include the Platonic and Archimedean solids.

Definition 3.1 (A family of soap-film-like surfaces with enclosed volume). Let $\Gamma$ be a convex uniform polytope of dimension $m \leq n$ of unit edge length embedded in $\mathbb{R}^{n}$ and centered at the origin. Let $\mathbf{p}_{1}, \ldots, \mathbf{p}_{k}$ be the vertices of $\Gamma$. Then $\sum_{i} \mathbf{p}_{i}=\mathbf{0}$ and $\left\|\mathbf{p}_{i}-\mathbf{p}_{j}\right\|=1$ for all vertices $\mathbf{p}_{i}$ and $\mathbf{p}_{j}$ that are adjacent in $\Gamma$.

For each $\mathbf{p}_{i}$ let $C_{i}$ be the set of points of $\mathbb{R}^{n}$ lying strictly closer to $\mathbf{p}_{i}$ than to any other $\mathbf{p}_{j}$, and define $K$ to be the complement of $\bigcup C_{i}$ (if $k=1$, let $K=\emptyset$ ). We note then that $C_{i}$ and $C_{j}$ share boundary non-trivially (on a set of non-zero $\mathcal{H}^{n-1}$ measure) if and only if $\mathbf{p}_{i}$ and $\mathbf{p}_{j}$ are adjacent vertices in $\Gamma$.

Next, choose $r \geq 0$ and define $B$ as the intersection of the open balls of radius $r$ centered at $-r \mathbf{p}_{i}$. Let $S$ be the boundary of $B$ and let $\beta$ be the volume of $B$.

Finally, define

$$
M=M(\Gamma, r)=(K \backslash \bar{B}) \cup S .
$$

Figure 1 contains examples of $M$ for various polytopes $\Gamma$. We note that in our construction the faces of $B$ correspond to the vertices of $\Gamma$ and the vertices of $B$ correspond to the faces of $\Gamma$. Thus when this construction is applied to a uniform polytope $\Gamma$, the bubble $B$ will be homeomorphic to the dual polytope of $\Gamma$. This is why the bubble resulting from $\Gamma=$ an icosahedron in Figure 1 below looks like a dodecagon.

Remark 3.2. As $\left\|\mathbf{p}_{i}\right\|=\left\|\mathbf{p}_{j}\right\|$ for all $i, j$, we see that $B=\emptyset$ if and only if $\left\|\mathbf{p}_{i}\right\|<1$ or $r=0$. If this is the case, $M=K$ and any volume constraint is vacuous. The proof below will apply to either case. Convex regular polytopes for which $\left\|\mathbf{p}_{i}\right\|<1$ and for which this construction may yield a non-empty bubble $B$ include: $(m=2)$ $k$-gons with $k<6,(m=3)$ all platonic solids except the regular dodecahedron, and $(m \geq 4)$ the $m$-simplex and the $m$-orthoplex. Minimizers over convex uniform polytopes such that $\left\|\mathbf{p}_{i}\right\| \geq 1$ also exist, but need not be convex or have spherical caps as faces.

\section{The Minimization theorem}

Theorem 4.1. Given $\Gamma$ and $r \geq 0$, the surface $M=M(\Gamma, r)$ is area-minimizing in the following sense.

Let $L_{0}$ be the closure of a bounded open set in $\mathbb{R}^{n}$ such that $M_{0}=M \cap L_{0}$ contains all of $S$. Let $T$ be any compact surface (rectifiable set) such that

$$
\left(M \backslash M_{0}\right) \cup T
$$

divides $\mathbb{R}^{n}$ into $k$ unbounded components $T_{i}$ such that $C_{i} \backslash L_{0} \subset T_{i}$ and $T_{i}$ and $T_{j}$ share boundary non-trivially only if $\mathbf{p}_{i}$ is adjacent to $\mathbf{p}_{j}$ in $\Gamma$. Let $U$ be the union 


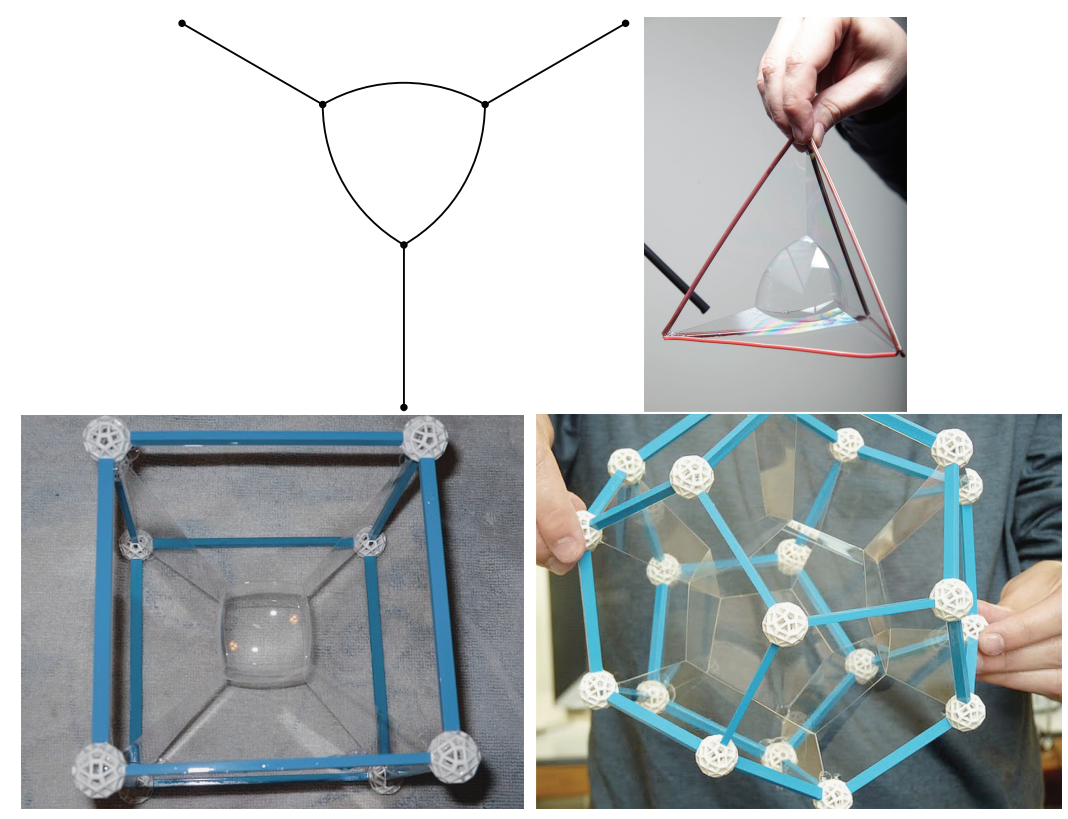

Figure 1. Examples of $M$ for $\Gamma=$ an equilateral triangle, a regular tetrahedron, a regular octahedron, a regular icosahedron

of the compact components, and suppose that the volume of $U$ is $\beta$, the same as the volume of $B$. Then

$$
\mathcal{H}^{n-1} M_{0} \leq \mathcal{H}^{n-1} T \text {. }
$$

Proof. Take an arbitrary $L_{0}$ and define $M_{0}$ as in the statement of the theorem. Choose a competing surface $T$.

Label the unbounded components of $\left[\left(M \backslash M_{0}\right) \cup T\right]^{C}$ as $T_{1}, \ldots, T_{k}$ and label the union of the compact components as $U$, as in the statement of the theorem. For each $i$ let

and for each $i \neq j$ let

$$
X_{i}=\partial U \cap \overline{T_{i}},
$$

$$
W_{i j}=\left(\partial T_{i}\right) \cap\left(\partial T_{j}\right) \cap L_{0} .
$$

Note then that $\mathcal{H}^{n-1} W_{i j} \neq 0$ only if $\mathbf{p}_{i}$ and $\mathbf{p}_{j}$ are adjacent in $\Gamma$. This labeling is illustrated in an example in Figure 2 .

For each point $\mathbf{p}_{i}$ define a constant vector field $\mathbf{v}_{i}(\mathbf{x})=\mathbf{p}_{i}$.

Use the Knothe-Gromov approach to define a volume-preserving map $F$ from $U$ to the convex set $B$, with $\operatorname{div} F \geq n$ wherever defined. Think of the map $\frac{1}{r} F$ as a vector field on $U$, so that

$$
\operatorname{div} \frac{1}{r} F \geq \frac{n}{r}
$$

Notice now that every point $\mathbf{y} \in B$ is within distance $r$ of $-r \mathbf{p}_{i}$ for all $1 \leq i \leq k$, which implies that $\left\|\frac{1}{r} \mathbf{y}+\mathbf{p}_{i}\right\| \leq 1$. So we see that the sum of vectors $\frac{1}{r} F+\mathbf{v}_{i}$ has length at most 1 anywhere. Recall that the difference vectors $\mathbf{v}_{i}-\mathbf{v}_{j}=\mathbf{p}_{i}-\mathbf{p}_{j}$ are also of length 1 on $W_{i j}$ if $W_{i j}$ has non-zero measure. From this point on, we ignore all $W_{i j}$ of measure zero. Let us establish the convention that the (unit) normal 


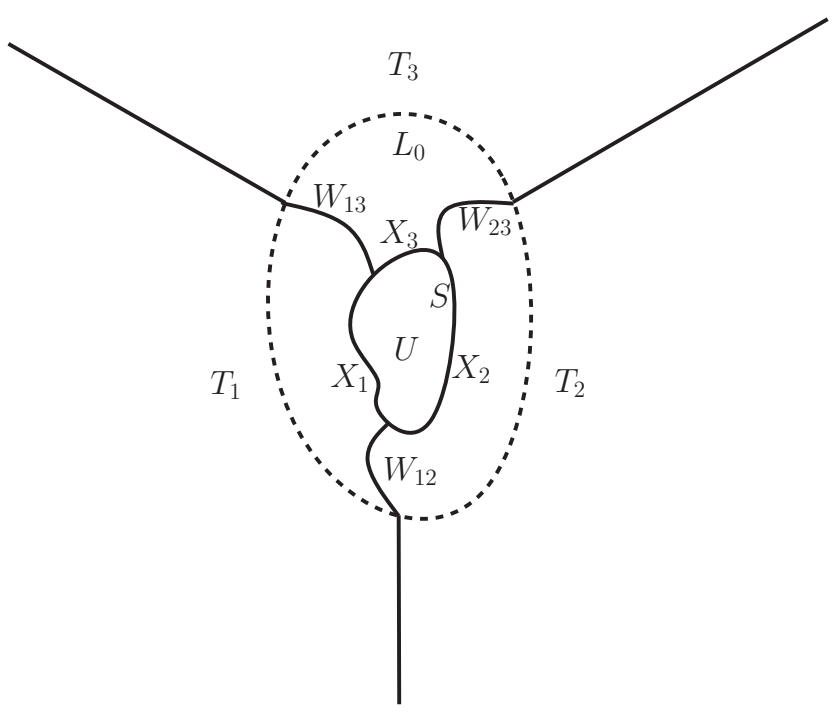

FiguRE 2. Illustration of labeling $(n=2, k=3)$

$\mathbf{n}_{0}$ to $\partial U$ is outward pointing, and the normal $\mathbf{n}_{i j}$ to $W_{i j}$ points toward the region with lower index. We may now compute:

$$
\begin{aligned}
\mathcal{H}^{n-1} T & =\int_{\partial U} 1+\sum_{i<j} \int_{W_{i j}} 1 \\
& \geq \sum_{i} \int_{X_{i}}\left(\frac{1}{r} F+\mathbf{v}_{i}\right) \cdot \mathbf{n}_{0}+\sum_{i<j} \int_{W_{i j}}\left(\mathbf{v}_{i}-\mathbf{v}_{j}\right) \cdot \mathbf{n}_{i j} .
\end{aligned}
$$

We wish to rewrite (2) by considering the flux into the region $T_{i}$ due to $\mathbf{v}_{i}$. This is the flux through $\partial T_{i} \cap T=\left(\bigcup_{i} W_{i j}\right) \cup X_{i}$, with normal $\mathbf{n}_{i}$ pointing into $T_{i}$. From the first sum we have the contribution of $\mathbf{v}_{i}$ through $X_{i}$. For each $j$ we also have flux through $W_{i j}$ from the second sum. Notice that if $i<j$, then $\mathbf{n}_{i j}=\mathbf{n}_{i}$, while if $i>j$, then $\mathbf{n}_{i j}=-\mathbf{n}_{i}$. Thus we may rewrite (2) as

$$
\begin{aligned}
& \int_{\partial U} \frac{1}{r} F \cdot \mathbf{n}_{0}+\sum_{i} \int_{\partial T_{i} \cap T} \mathbf{v}_{i} \cdot \mathbf{n}_{i} \\
= & \int_{U} \operatorname{div} \frac{1}{r} F+\sum_{i} \int_{\partial T_{i} \cap T} \mathbf{v}_{i} \cdot \mathbf{n}_{i} \\
\geq & \frac{n}{r} \operatorname{Volume}(U)+\sum_{i} \int_{\partial T_{i} \cap T} \mathbf{v}_{i} \cdot \mathbf{n}_{i} .
\end{aligned}
$$

Notice that any term in the sum in (5) is the flux of a constant vector field through a surface with fixed boundary. We see then that the quantity (5) is independent of $T$ (with $L_{0}$ held constant).

We claim that equality holds throughout when we apply this estimate to the conjectured minimal surface. When $T=M_{0}$, we have $F(\mathbf{x})=\mathbf{x}$. The inward pointing unit normal to $X_{i}$ at $\mathbf{x}$ is given by $\frac{1}{r} \mathbf{x}+\mathbf{p}_{i}=\frac{1}{r} F(\mathbf{x})+\mathbf{v}_{i}$. Also, notice 
that $W_{i j}$ consists of points equidistant from $\mathbf{p}_{i}$ and $\mathbf{p}_{j}$, so if $i<j$, the normal to $W_{i j}$ is given by $\mathbf{p}_{i}-\mathbf{p}_{j}$. This shows that we have equality from (11) to (2). Also notice that if $F$ is the identity function, then $\operatorname{div} F=n$, giving us equality in the last step (5).

Letting $G(T)=\sum_{i} \int_{X_{i}}\left(\frac{1}{r} F+\mathbf{v}_{i}\right) \cdot \mathbf{n}_{0}+\sum_{i<j} \int_{W_{i j}}\left(\mathbf{v}_{i}-\mathbf{v}_{j}\right) \cdot \mathbf{n}_{i j}$ from (2) above, we see that this argument reduces to a proof that

$$
\mathcal{H}^{n-1} T \geq G(T) \geq G\left(M_{0}\right)=\mathcal{H}^{n-1} M_{0} .
$$

We have shown that $M_{0}$ is mass minimizing among all figures that contain the same volume, have the same boundary, and have the same connectivity (regions $T_{i}$ and $T_{j}$ for some competitor $T$ share boundary non-trivially only if they do in $M_{0}$ ). If $\Gamma$ is a regular simplex then, as $\Gamma$ is also a complete graph, $T_{i}$ shares non-trivial boundary in $M_{0}$ with all other $T_{j}$. Thus we have the following corollary:

Corollary 4.2. If $\Gamma$ is a regular simplex, then $M_{0}$ is minimizing as above but without the connectivity condition.

It is an open problem however as to which figures $M(\Gamma, r)$ other than those generated from a regular simplex are minimizing in this more general setting.

Corollary 4.3. If $\Gamma^{\prime}$ is the dual polytope to some convex uniform polytope $\Gamma$, then the cone over $\Gamma^{\prime}$ is minimizing among all other surfaces that share the same boundary and have the same connectivity.

Proof. Apply Theorem 4.1 to $M(\Gamma, 0)$.

The above corollary is a stronger version of the main result of [3], which showed that stationary cones are minimizing over diffeomorphisms. Choe's results however apply to the more general class of polyhedral sets, and not just cones.

\section{Metacalibration}

The proof of Theorem 4.1 is an example of a method which we call metacalibration. Calibration arguments [6] typically have the form

$$
\mathcal{H}^{n} M=\int_{M} \varphi=\int_{M^{\prime}} \varphi \leq \mathcal{H}^{n} M^{\prime}
$$

for some particular differential form $\varphi$, showing that $M$ is mass minimizing among all competitors $M^{\prime}$. This method has been useful in solving fixed boundary problems, such as Plateau's problem (see 9], cf. Chapter 6). Metacalibration generalizes the traditional calibration argument by allowing $\varphi$ to vary for each competitor. This allows metacalibration to tackle problems with fixed volume constraints. As demonstrated in this paper, metacalibration can also handle combinations of fixed boundary and fixed volume constraints.

Using a variation of the Knothe-Rosenblatt rearrangement in a metacalibration argument, the authors were able to prove the double bubble conjecture in the plane [4. Optimal transport promises to be another tool for constructing metacalibrations. For instance, the Brenier map [1] has many of the same properties as the Knothe-Rosenblatt rearrangement, including the divergence criterion used in this paper. We hope that a suitable generalization of the Brenier map can be used to solve other isoperimetric problems such as the triple bubble conjecture. 


\section{REFERENCES}

[1] Yann Brenier. Polar factorization and monotone rearrangement of vector-valued functions. Comm. on Pure and Applied Math., 44:375-417, 1991. MR.1100809 (92d:46088)

[2] John Brothers and Frank Morgan. The isoperimetric theorem for general integrands. Michigan Math. J., 41(3):419-431, 1994. MR.1297699 (95g:49080)

[3] Jaigyoung Choe. Every stationary polyhedral set in $R^{n}$ is area minimizing under diffeomorphisms. Pacific J. Math., 175(2):439-446, 1996. MR1432839 (98e:49094)

[4] Rebecca Dorff, Gary Lawlor, Donald Sampson, and Brandon Wilson. Proof of the planar double bubble conjecture using metacalibration methods. Involve, 2(5):611-628, 2009. MR2601581

[5] Michael Gromov. Isoperimetric inequalities in Riemannian manifolds, Appendix I to Asymptotic Theory of Finite Dimensional Normed Spaces by Vitali D. Milman and Gideon Schechtman, Lecture Notes in Mathematics, No. 1200. Springer-Verlag, New York, 1986. MR0856576 (87m:46038)

[6] Reese Harvey and H. Blaine Lawson Jr. Calibrated geometries. Acta Mathematica, 148(1) (1982), 47-157. MR0666108 (85i:53058)

[7] Herbert Knothe. Contributions to the theory of convex bodies. Michigan Math. J., (4):39-52, 1957. MR0083759(18:757b)

[8] Gary R. Lawlor and Frank Morgan. Paired calibrations applied to soap films, immiscible fluids, and surfaces or networks minimizing other norms. Pacific J. Math., 166(1):55-83, 1994. MR1306034 (95i:58051)

[9] Frank Morgan. Geometric Measure Theory: A Beginner's Guide. Academic Press, 1988. MR933756 (89f:49036)

[10] Murry Rosenblatt. Remarks on a multivariate transformation. Ann. Math. Statist., 23(3):470472, 1952. MR0049525(14:189j)

[11] Jean Taylor. The structure of singularities in soap-bubble-like and soap-film-like minimal surfaces. Annals of Math., 103:489-539, 1976. MR0428181 (55:1208a)

Department of Mathematics, Brigham Young University, Provo, Utah 84604

E-mail address: beccadorff@gmail.com

Department of Mathematics, Brigham Young University, Provo, Utah 84604

E-mail address: werd2.718@gmail.com

Department of Mathematics, Brigham Young University, Provo, Utah 84604

E-mail address: lawlor@mathed.byu.edu

Department of Mathematics, Brigham Young University, Provo, Utah 84604

E-mail address: sampson.dcs@gmail.com 